\title{
Performance Evaluation of DSRC-Zigbee Heterogeneous Network for Intelligent Transportation System
}

\author{
Randi Eriko Hariyadi*, E. Endroyono, Achmad Affandi \\ Departement of Electrical Engineering \\ Institut Teknologi Sepuluh Nopember \\ Kampus ITS, Keputih \\ Surabaya, Indonesia
}

\begin{abstract}
Transportation is one of the primary needs of human beings that cannot be avoided, with the increasing vehicle can lead to a congested road situation which can lead to less safe road safety. An Intelligent Transportation System (ITS) can be used to increase road safety. This system uses a Dedicated Short-Range Communication (DSRC) protocol for network access due to its low latency transmission time. Unfortunately, there is research shows that DSRC has a performance issue in a dense area or increased network load. This problem is mostly solved with a heterogeneous network DSRC-LTE but utilizes mobile phone networks that dynamically change can lead to inconsistent and unpredictable network performance. There is some research about ZigBee for ITS shows that it is decent enough for non-critical applications. Thus, the authors try to utilize Zigbee to create the DSRC-Zigbee heterogeneous network so that the network is independent for the ITS application. The proposed heterogeneous network is a fixed model due to its simple architecture. OMNeT++ and Simulation of Urban Mobility (SUMO) are used to evaluate the performance of the network in an urban area with various scenarios. The simulation result shows that the proposed heterogeneous network is capable to improve the messages dissemination rate by $15.78 \%$ and $1.22 \%$ in a certain scenario compared to the homogeneous network DSRC only.
\end{abstract}

Keywords: Zigbee, DSRC, Heterogeneous Network, Simulation, OMNeT++, SUMO

\section{INTRODUCTION}

Intelligent Transportation System (ITS) is one of the smart city sub-systems which aims to the road safety and road traffic efficiency improvement. One of the methods to achieve the goals is to deploy a vehicle to vehicle (V2V) communication so that every vehicle can exchange data to be processed and convert it to useful information for the driver. For this kind of environment, Dedicated Short Range Communication (DSRC)/IEEE $802.11 \mathrm{p}$ is the main candidate as this particular technology is designed for $\mathrm{V} 2 \mathrm{~V}$ as it has low latency transmission time and high bandwidth. Unfortunately, research in [1], [2] shows that DSRC performance is highly degraded in a dense environment or increased load. To overcome this problem, a heterogeneous network is a major choice.

A heterogeneous network is a combination of different radio access technology that complement each other to serve a differentiated service requirement [3] There are two kinds of heterogeneous network. First is the fixed architecture, which means the network interface is predefined and do not change over time, and second is the dynamic architecture which can adapt to the network condition. In [1] a survey about fixed and dynamic architecture shows that the dynamic network may cause an unstable network due to maintaining node connection in a highly dynamic vehicular network, which is a major

\footnotetext{
* Corresponding Author.

Email: randi.eriko@live.com

Received: June 24, 2021

Accepted: August 17, 2021

Open access under CC-BY-NC-SA

(C) 2021 PPET - LIPI
}

; Revised: July 27, 2021

; Published: August 31, 2021 issue to be considered. On the other hand, fixed architecture provides a simple and time-invariant architecture, the choice of which network interface to use should be based on the message type. The drawback of this fixed architecture is the lack of flexibility.

Deploying either fixed or dynamic heterogeneous network is capable to increase overall network performance as shown in [3], [4] that utilize LTE and DSRC. But in some cases, LTE network is utilized for an in-car entertainment system which nowadays smartphone and mobile devices are sufficient to serve an entertainment purpose. And also, sharing network resources for the ITS can lead to an unpredictable network load as mobile phone usage is an uncontrollable variable. Because of this reason, it can lead to unpredictable network performance for the ITSs network. Thus, the author tries to use another radio technology that is widely available, can serve the ITS network independently, and has a low deployment cost.

Zigbee/IEEE802.15.4 is deemed as one of the best candidates to start with. A few research shows that Zigbee performance is promising. In research done by Tytgat et al. [5] investigating the performance of Coexistence Aware Clear Channel Assessment (CACCA) protocol for Zigbee and Wi-Fi. The result is the packet error rate is decreased by $24 \%$ if deployed on Zigbee only, and decreased by $75 \%$ if deployed on Wi-Fi only, and decreased $99.6 \%$ on both Wi-Fi and Zigbee. With this parameter, it's conducted that the Zigbee is compliant with the ITS standard stated in [6].

In [7], Zigbee performance is evaluated in an urban area look-alike scenario, where another Wi-Fi network is present. Evaluation is on channel 26 because it is the 
farthest channel overlap with Wi-Fi system. This study shows that Zigbee network is only affected while there is channel 13 of Wi-Fi nearby. The average end-to-end delay is about $200 \mathrm{~ms}$ for sending 1024 bits messages. Similar results are shown in [8] where the end-to-end delay for the Zigbee network is for about $150 \mathrm{~ms}$ to $200 \mathrm{~ms}$. With such delay, it is deemed quite high to be used for ITS. In conclusion, Zigbee alone may not be suitable for some time critical ITS application.

Meanwhile, DSRC is capable of low latency transmission but suffers in a dense area or increased network load, Zigbee provides a reasonable performance to be used in a non-critical scenario. Thus, we combine both of these radio access into one system to serve different types of services to split the load in the DSRC network.

In this paper, we proposed a fixed architecture of a heterogeneous network consisting of DSRC and Zigbee for the V2V ITS application due to its simple architecture. The network performance will be evaluated by using an OMNeT++ simulator combined with Simulation of Urban Mobility (SUMO) to simulate the traffic to obtain a real-world model. There will be various payload sizes and propagation environments to evaluate how the response of the network to the increased network load and propagation environment.

\section{Proposed Model}

In this study, fixed heterogeneous network architecture is proposed as shown in Figure 1. The proposed heterogeneous network model is generating messages based on the message type consisting of Road Safety and Other app type. Each generated message will be labeled with either of these types. For every message generation, it will pass to the selector to select which radio access is to be used. The radio access selector is using a simple algorithm, as shown in Figure 2. It decides the radio access for each message based on the label. The road safety message that aims to reduce road accidents by sending Cooperative Awareness Messages (CAM) in a certain interval, must be sent through the network with the lowest latency possible, hence the DSRC is used. On the other hand, the other app that doesn't require a low latency is sent through Zigbee.

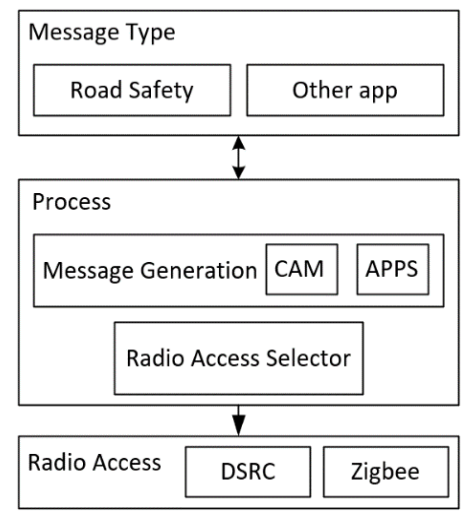

Figure 1. Fixed Heterogeneous Network Architecture.

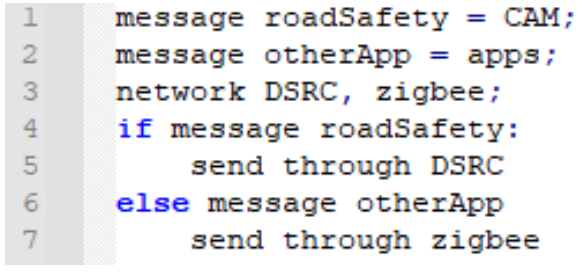

Figure 2. Pseudocode of Radio Access Selector.

There are two message types. First is the Road safety message with CAM and second is the other application type that can be used for any purpose with other app messages. In this paper, all payload that sent through the network is a dummy payload, just to represent actual of usable data as our main scope is the network performance. The sent interval for both messages is set to $1 \mathrm{~Hz}$, and single hop only according to ITS standard as specified [9].

CAM is sent through the DSRC/IEEE 802.11p frame, with the following frame models as shows in Figure 3. While transmitted through the air, the total size to be sent is 63 bytes consist of 5 bytes OFDM header, 802.11 MAC header, 2 bytes ether type, 20 bytes IP header, 8 bytes UDP header, and 4 bytes MAC trailer, plus the additional usable payload up to 1442 byte, as the maximum IEEE 802.11 frame is 1500 bytes. While other app sent through IEEE 802.15.4 frame is consist of 802.15.4 9 bytes MAC header, 20 bytes IP Header, and 8 bytes UDP header, plus the additional payload up to 90 bytes as shown in Figure 4. In short, payload terms in this paper mean the actual usable data, not to be confused with the total full frame size.

The size of the payload does not have a standardization yet, thus the author refers to the previous research by Shen, et al. [3], as the CAM size is 400 bytes. While the other application is set to 90 bytes, as this the maximum IEEE 802.15.4 frame size, 200 bytes, and 400 bytes to set the size as big as half and same size of the CAM. In short, there are three kinds of messages payload. First 400 bytes CAM +90 bytes other app, second 400 bytes CAM +200 bytes other app, and third 400 bytes CAM +400 bytes another app.

\section{Simulation SETUP}

There are two scenarios will be used for the evaluation. The first scenario is to send CAM and another app message through a DSRC homogeneous network. The second scenario is to send CAM and other app messages through the proposed fixed heterogeneous network architecture which each message is sent through different radio access.

\begin{tabular}{|c|c|c|c|c|c|c|}
\hline $\begin{array}{c}\text { OFDM } \\
\text { PHY } \\
\text { HEADER } \\
\text { (5 B) }\end{array}$ & $\begin{array}{c}802.11 \text { MAC } \\
\text { HEADER (24 B) }\end{array}$ & $\begin{array}{c}\text { ETHER } \\
\text { TYPE } \\
\text { (2 B) }\end{array}$ & $\begin{array}{c}\text { IP HEADER } \\
\text { (20 B) }\end{array}$ & $\begin{array}{c}\text { UDP } \\
\text { HEADER } \\
\text { (8 B) }\end{array}$ & PAYLOAD (0-1442 B) & $\begin{array}{c}802.11 \\
\text { MAC } \\
\text { TRAILER } \\
\text { (4 B) }\end{array}$ \\
\hline
\end{tabular}

Figure 3. CAM frame model.

\begin{tabular}{|c|c|c|c|}
\hline $\begin{array}{c}802.15 .4 \\
\text { MAC HEADER } \\
\text { (9 B) }\end{array}$ & IP HEADER (20 B) & $\begin{array}{c}\text { UDP } \\
\text { HEADER } \\
\text { (8 B })\end{array}$ & PAYLOAD (0-90 B) \\
\hline
\end{tabular}

Figure 4. Other App frame model. 


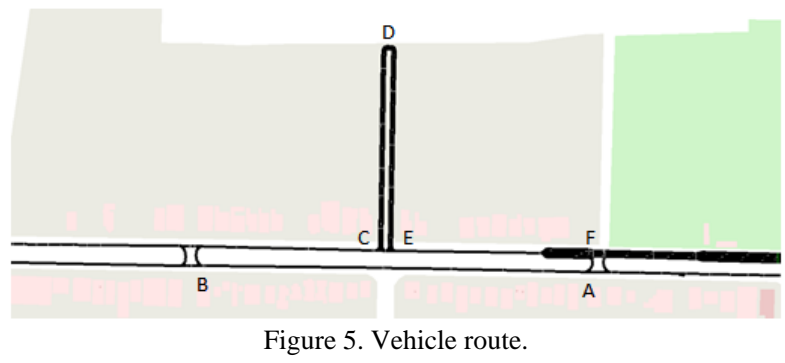

\section{A. Simulation Parameter}

The simulation will be run five times for each scenario to obtain more accurate results. Each run is one minute length with maximum of 35 vehicles in the urban area. Research by Imandita in [10] shows maximum number of vehicles that passes on certain area for oneminute window are 35 vehicles. Simulation area is using a real-world road model that shown in the Figure 5, where all vehicles are spread out evenly and follows the A-BC-D-E-F-A route.

Transceiver specification in the vehicle model in the simulation is set based on the real-world available transceiver for each corresponding technology. For the DSRC network interface, we set the parameter of Txpower to $-3 \mathrm{dBm}$, and Rx-sensitivity to $-97 \mathrm{dBm}$ according to [9]. While the Zigbee/802.15.4 we set the Tx Power to $0 \mathrm{dBm}$, and $\mathrm{Rx}$ sensitivity to $-101 \mathrm{dBm}$ according to [10]. For the frequency allocation, we use channel 26 of the Zigbee $(2680 \mathrm{MHz})$, and channel 174 of DSRC (5870 MHz). The antenna for each network interface is placed on the vehicle roof. And the antenna type is a monopole antenna with a $3 \mathrm{~dB}$ gain for each antenna as shows in Figure 6.

For the propagation model, we use the Rician Fading which is suitable to the $\mathrm{V} 2 \mathrm{~V}$ environment in which a multipath phenomenon occurs. Generally, Rician K factor describes how strong is the main signal in the Line of Sight (LOS) component. With the bigger K factor, the LOS component is stronger [11]. The Rician fading $\mathrm{K}$ factor is based on the research done by [12], [13] which each of them is using a real-world captured data measurement to estimate the Rician $\mathrm{K}$ factor. The results show that in the urban environment, typically ranged between $-5 \mathrm{~dB}$ to $10 \mathrm{~dB}$ with the Probability Density Function as shown in Figure 7 . The $\mathrm{K}$ factor is selected to be $-2 \mathrm{~dB}, 3 \mathrm{~dB}$, and $6 \mathrm{~dB}$ for each message type.

From the simulation, we get a dataset from $\mathrm{K}$ factor $-2 \mathrm{~dB}, 3 \mathrm{~dB}$, and $6 \mathrm{~dB}$ with total payload of 490 bytes, 600 bytes, and 800 bytes form homogeneous network and the heterogeneous network. Table 1 is the summary of the simulation parameter for each scenario.

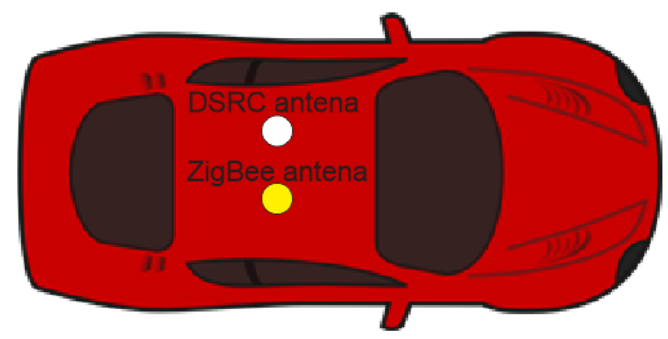

Figure 6. Vehicle ilustration

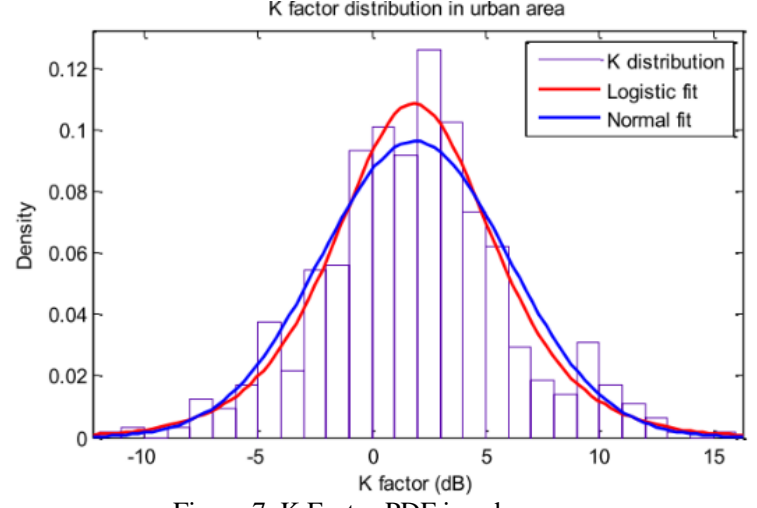

Figure 7. K Factor PDF in urban area.

\section{B. Performance Metrics}

For performance evaluation, three performance metrics are considered based on the study in [14], with slight modification. First is the end-to-end delay which counted start from the message generated until the message is processed and sent to the application layer, refer to (1). The second metric is the Packet Receive Ratio (PRR) which define as how many successful received packets are compared to the total packet sent by all vehicles in a one-time window, refer to (2). The third metric is the Packet Drop Ratio (PDR) which defines how many packets is failed to receive while the vehicle is on the communication range in a one-time window, refer to (3).

$$
\begin{gathered}
\text { delay }=\text { received time stamp }- \text { sent time stamp } \\
P R R=\frac{\text { received packet }}{\text { total packet sent }} \times 100 \% \\
P D R=\frac{\text { dropped packet }}{\text { total packet sent }} \times 100 \%
\end{gathered}
$$

Each of these metrics will be evaluated on each vehicle. The homogeneous and heterogeneous networks from the first and second scenarios will be compared with the same $\mathrm{K}$ factor and payload in every evaluation parameter. From this comparison, how the DSRC-Zigbee heterogeneous network performance compared to the

\begin{tabular}{|c|c|c|}
\hline Parameter & \multicolumn{2}{|c|}{ Value } \\
\hline Simulation Time & \multicolumn{2}{|l|}{$60 \mathrm{~s}$} \\
\hline Iteration & \multicolumn{2}{|c|}{$\begin{array}{l}5 \text { (for each combination } \mathrm{K} \text { factor } \& \\
\text { total payload value) }\end{array}$} \\
\hline Road Model & \multicolumn{2}{|c|}{ Real world scenario } \\
\hline Vehicle density & \multicolumn{2}{|l|}{35} \\
\hline Vehicle velocity & \multicolumn{2}{|c|}{$40 \mathrm{~km} / \mathrm{h}$ - Constant } \\
\hline Network & DSRC & Zigbee \\
\hline Frequency & $5870 \mathrm{MHz}$ & $2680 \mathrm{MHz}$ \\
\hline Transmission Power & $-3 \mathrm{dBm}$ & $0 \mathrm{dBm}$ \\
\hline Receiver Sensitivity & $-97 \mathrm{dBm}$ & $-101 \mathrm{dBm}$ \\
\hline \multicolumn{3}{|c|}{ Message Traffic } \\
\hline CAM Message Size & \multicolumn{2}{|l|}{400 Bytes } \\
\hline CAM send interval & \multicolumn{2}{|c|}{$1 \mathrm{~Hz}-$ Exact same time } \\
\hline App Message Size & \multicolumn{2}{|c|}{90 Bytes, 200 Bytes, 400 Bytes } \\
\hline App send interval & \multicolumn{2}{|c|}{$1 \mathrm{~Hz}$ - Exact same time } \\
\hline \multicolumn{3}{|c|}{ Propagation } \\
\hline K Factor & \multicolumn{2}{|c|}{$-2 \mathrm{~dB}, 3 \mathrm{~dB}, 6 \mathrm{~dB}$} \\
\hline
\end{tabular}
homogeneous network can be evaluated.

TABLE 1. SIMULATION PARAMETER 


\section{Simulation ReSults ANd ANALYSIS}

Simulation results will be presented in each evaluation parameter and each message payload scheme. A graphical comparison will be also provided, with blue line indicate the proposed fixed heterogeneous network, and orange is the homogeneous DSRC network.

\section{A. Delay}

\section{1) 400+90 bytes scheme}

While deploying a heterogeneous network, the delay required for Zigbee to send 90 bytes of other app messages is higher compared to the homogeneous network for $2.74 \mathrm{~ms}$. On the other hand, the delay required to send the road safety message is decreased by $0.24 \mathrm{~ms}$. The $\mathrm{K}$ factor in this scheme still did not shows any impact, as shows in Figure 8.

\section{2) 400+200 bytes scheme}

The delay difference for sending 200 bytes another app through Zigbee network compared to the homogeneous network DSRC is increased for $28.47 \mathrm{~ms}$ to $31.4 \mathrm{~ms}$. The $\mathrm{K}$ factor starts to show its impact on the Zigbee network as the delay decreases as the $\mathrm{K}$ factor value is bigger. Figure 9 shows on the 400 bytes CAM, by using the heterogeneous network the delay is just decreased by $0.53 \mathrm{~ms}$.

\section{3) 400+400 bytes scheme}

The delay needed for sending the other app 400 bytes is about $61.15 \mathrm{~ms}$ to $66.78 \mathrm{~ms}$. Compared to the homogeneous network, there is an increase for about $58.23 \mathrm{~ms}$ to $64.44 \mathrm{~ms}$. On the other side, the delay for sending road safety messages with a heterogeneous network is decreased by $1.06 \mathrm{~ms}$ is shown in Figure 10. In this scheme, the delay gets smaller as the $\mathrm{K}$ factor increase.
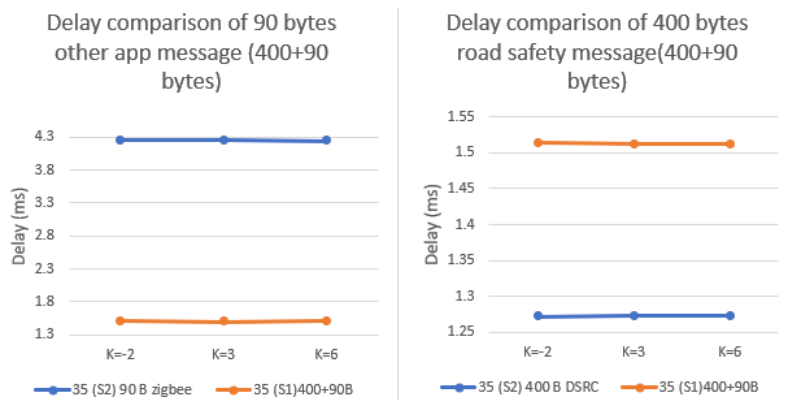

Figure 8 . Delay comparison for $400+90$ bytes scheme.

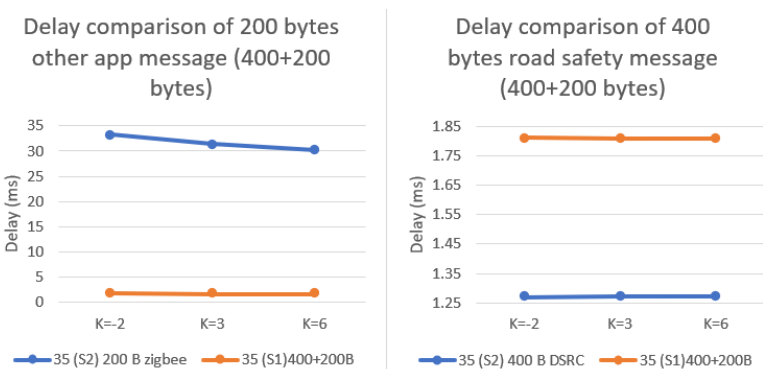

Figure 9. Delay comparison for $400+200$ bytes scheme.
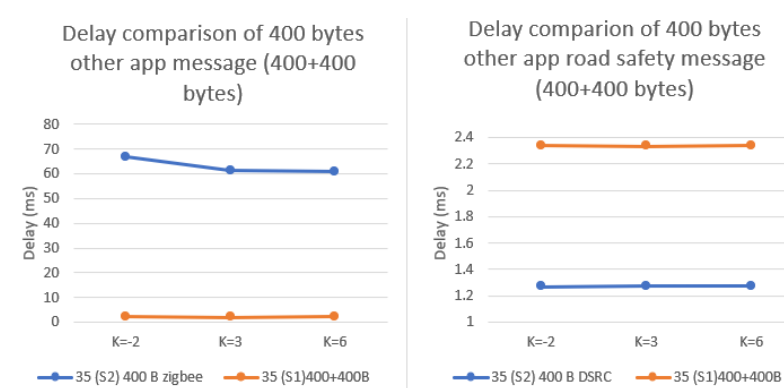

Figure 10. Delay comparison in $400+400$ bytes scheme.

\section{B. Packet Receive Ratio (PRR)}

\section{1) $400+90$ bytes Scheme}

The proposed heterogeneous network shows a big improvement in this payload scheme for the 90 bytes of other apps. While a homogeneous network is just capable to reach PRR for about $66.5 \%$ to $73.08 \%$, the heterogeneous network can reach $82.22 \%$ to $88.86 \%$. The improvement is $14.91 \%$ to $15.78 \%$, depends on the $\mathrm{K}$ factor value, as the higher $\mathrm{K}$ factor yield a better result. But for the 400 bytes road safety, there is just a slight improvement for the PRR, just about $0.27 \%$ to $1.22 \%$ as shows in Figure 11.

\section{2) $400+200$ bytes scheme}

Figure 12 is the bigger payload shows that the proposed heterogeneous network shows a big performance drop. To send the 200 bytes of other app in the Zigbee network, the PRR is only $27.88 \%$ to $35.75 \%$, which if compared to the homogeneous network is $64.36 \%$ to $72.55 \%$. It means the PRR is dropped $36.47 \%$ to $38.81 \%$. While the improvement of the road safety message is only $1.07 \%$ to $2.41 \%$. Same as before, the K factor influence shows that the bigger the $\mathrm{K}$ factor value, the difference is getting smaller.
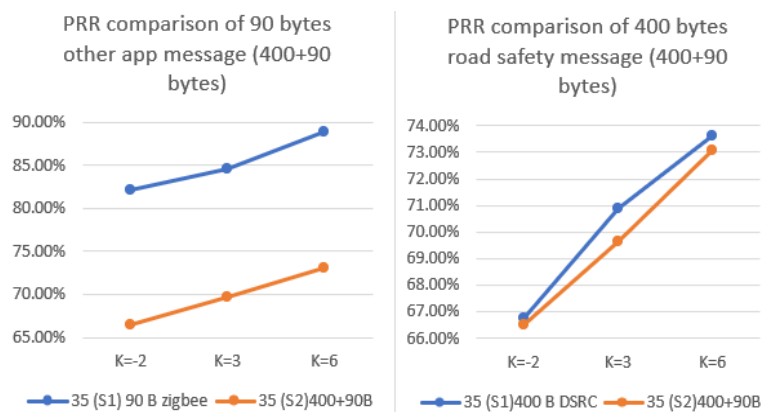

Figure 11. PRR comparison for $400+90$ bytes scheme.
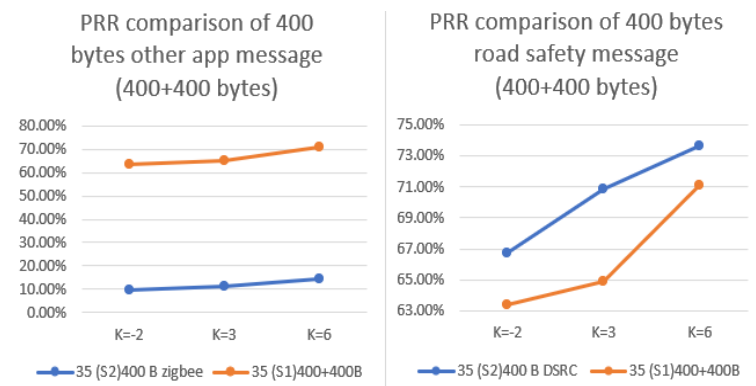

Figure 12. PRR comparison for $400+200$ bytes scheme. 


\section{3) $400+400$ bytes scheme}

In this scheme, the PRR of other app 400 bytes sent through the heterogeneous network using Zigbee also have a severe loss. The PRR is only $9.88 \%$ to $14.23 \%$ which compared to the homogeneous network has a $53.74 \%$ to $56.89 \%$ difference. For the 400 bytes road safety message, the heterogeneous network is improving the PRR for $2.5 \%$ to $5.94 \%$, as shows in Figure 13. As we investigate this huge performance loss, it is caused by fragmentation in the bigger payload scheme. The Carrier Sense Multiple Access - Collision Avoidance (CSMACA) mechanism could not keep up with the queue of the packet to be sent as there are many vehicles that must send the next update within one second as ITS minimal requirement, resulting in a packet loss.

\section{Packet Drop Ratio (PDR)}

1) 400+90 bytes scheme

In the 90 bytes other app type message, the heterogeneous network has a bigger PDR compared to the homogeneous network with a $1.99 \%$ to $4.02 \%$ difference. In contrast, the 400 bytes road safety message homogeneous network has a bigger PDR compared to the heterogeneous network with a $0.22 \%$ to $1.1 \%$ difference. Figure 14 shows the $\mathrm{K}$ factor influence is also had a noticeable impact, where in the 90 bytes other app the difference is closing as the $\mathrm{K}$ factor is increased, but in the 400 bytes road safety, $K$ factor $3 \mathrm{~dB}$ have a slight difference compared to the other.

\section{2) 400+200 bytes scheme}

In this scheme, as we discovered that Zigbee is degraded so much in the higher payload size in the PRR comparison section. As expected, the PDR in this payload scheme also has a big difference. The 200 bytes other app message that sent through Zigbee in the heterogeneous network has a higher PDR is from $18.75 \%$
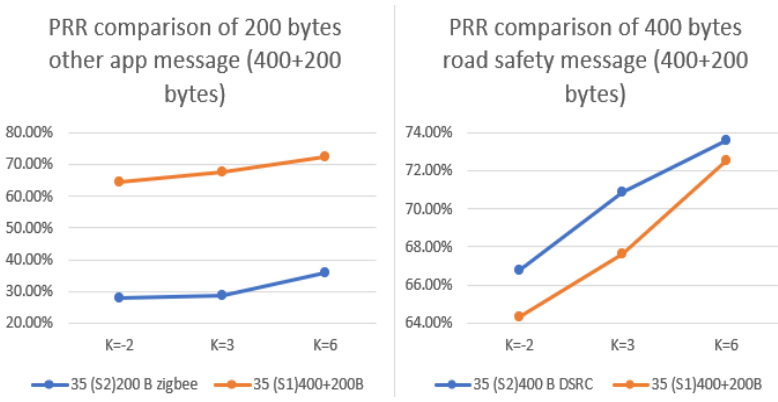

Figure 13. PRR comparison for $400+400$ bytes scheme.
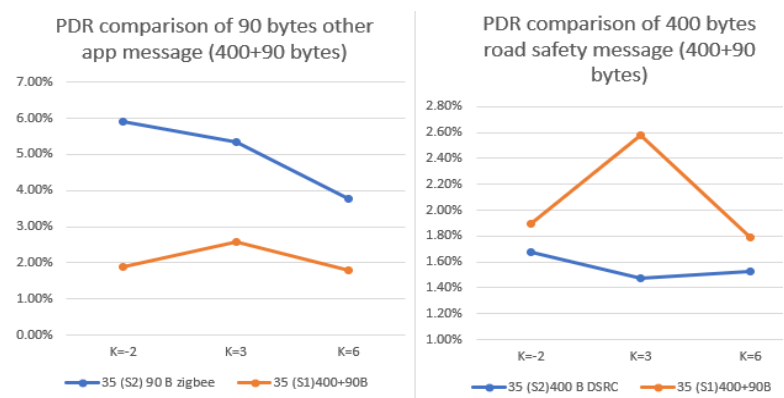

Figure 14. PDR comparison for $400+90$ bytes scheme. to $22.44 \%$ compared to the homogeneous network. While in 400 bytes road safety, the heterogeneous network had a smaller PDR compared to the homogeneous with $0.5 \%$ to $1.25 \%$ difference. The $\mathrm{K}$-factor influence shows that as $\mathrm{K}$ factor getting bigger, the difference is smaller as shown in Figure 15.

\section{3) 400+400 bytes scheme}

In this scheme, the decreased performance is quite small compared to the $400+200$ bytes scheme. In the other app 400 bytes message sent through the Zigbee network in the heterogeneous network has a bigger PDR than the homogeneous network for about $22.84 \%$ to $24.44 \%$. While the 400 bytes road safety messages, the heterogeneous network has a smaller PDR compared to the homogeneous network by $1.11 \%$ to $1.54 \%$ as shows in Figure 16.

The difference between $400+200$ bytes and $400+400$ bytes is not too different is because the network of the Zigbee is already highly saturated from the $400+200$ bytes scheme. Thus, an additional 200 bytes payload in $400+400$ bytes scheme didn't bring a major impact to the network performance. But if we see in the PRR section, there is a noticeable difference, this is due to the fragmented packet, as if there is only one fragment is dropped resulting the other successful reception is discarded due to incomplete packet. For example, if the packet fragmented to 4 fragments, all fragments need to be received to be considered as successful reception. On the other hand, even if only one fragment is dropped, it will be considered as dropped packet.

\section{Discussion}

\section{1) 400+90 bytes scheme}

In this scheme, the proposed heterogeneous network performance in the 90 bytes other app messages shows

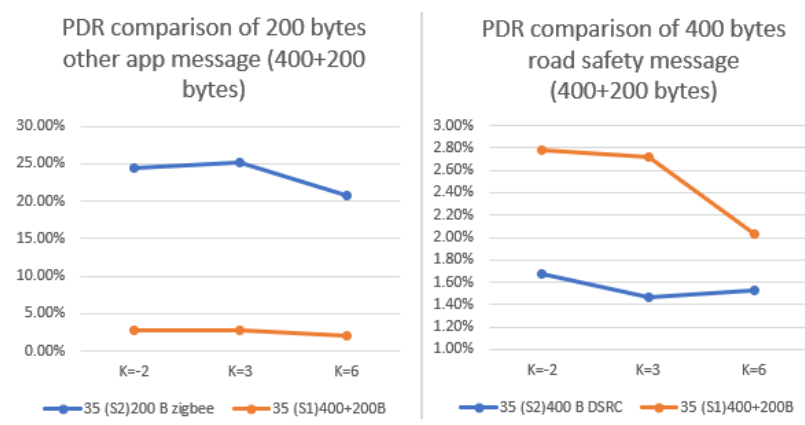

Figure 15 . PDR comparison for $400+200$ bytes scheme.
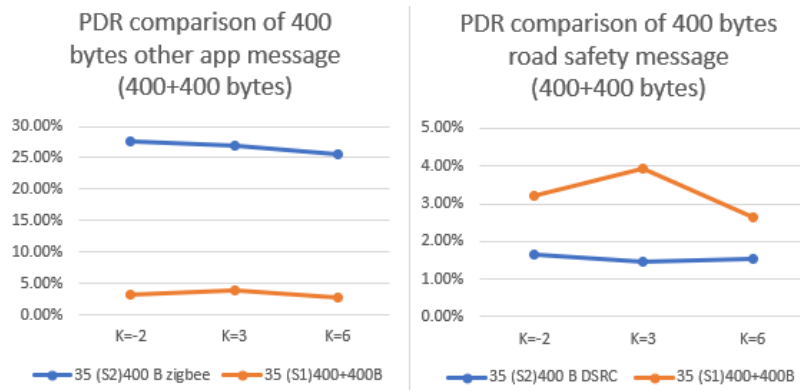

Figure 16. PDR comparison for $400+400$ bytes scheme. 
increased PDR. But the PRR parameter shows a big improvement, compared to the homogeneous network, that $2.74 \mathrm{~ms}$ increased delay, and $1.99 \%$ to $4.02 \%$ the PRR is increased by $14.91 \%$ to $15.78 \%$. The 400 byte road safety message shows a slight improvement in all parameters. The delay required for transmission is $0.24 \mathrm{~ms}$ lower, $0.27 \%$ to $1.22 \%$ increased PRR, and 0.22 to $1.1 \%$ lower PDR. In short, this scheme is capable to increase both road safety and other app overall performance.

\section{2) 400+200 bytes scheme}

In this scheme, the proposed heterogeneous network can only improve the road safety messages with a slight improvement. The delay is decreased by $0.53 \mathrm{~ms}, 1.07 \%$ to $2.41 \%$ increased PRR, and $0.5 \%$ to $1.25 \%$ decreased PDR. Such improvement is yielding a big performance drop in the other app messages with $28.47 \mathrm{~ms}$ to $31.4 \mathrm{~ms}$ increased delay, $36.47 \%$ to $38.81 \%$ lower PRR, and $18.75 \%$ to $22.44 \%$ increased PDR.

\section{3) 400+400 bytes scheme}

In this scheme, the proposed heterogeneous network is beneficial for the road safety messages only. It lowers the transmission delay for $1.06 \mathrm{~ms}, 2.5 \%$ to $5.94 \%$ better PRR, and $1.11 \%$ to $1.54 \%$ lower PDR. While the other app messages are highly degraded. The delay is increased by $58.23 \mathrm{~ms}$ to $64.44 \mathrm{~ms}, 18.75 \%$ to $22.44 \%$ lower PRR, and $22.84 \%$ to $24.44 \%$ higher PDR.

\section{4) $K$ factor}

The $\mathrm{K}$ factor impact on the delay parameter gives a small difference in each value. The $\mathrm{K}$ factor starts to show the influence in the PDR and PRR parameters. Which the bigger $\mathrm{K}$ factor yield a better result, we can see that in the PRR parameter, in all payloads scheme the results are getting higher as the $\mathrm{K}$ factor is increased. While in the PDR parameter, the higher $\mathrm{K}$ factor value has a smaller PDR.

\section{CONCLUSION}

In this paper, the proposed fixed heterogeneous network Zigbee-DSRC can slightly improve the dissemination of the CAM messages up to $1.22 \%$, while in other app messages, it reaches up to $15.78 \%$ improvement. This can be achieved if the Zigbee payload is not fragmented while being transmitted over the air.

The Zigbee CSMA-CA mechanism in higher fragment numbers with increased payload size in the ITS network scenario yields to a high end-to-end delay increase, up to $68 \mathrm{~ms}$ difference in the simulation result above. This is because of the fragment queue from all vehicles that sharing the same transmission medium transmitting within same time.

From the simulation result, the $\mathrm{K}$ factor had a noticeable impact with a difference up to $7 \%$ in PRR parameter for each different $\mathrm{K}$ factor value that being evaluated. Thus, for a more accurate result, we suggest using an actual world measurement and estimation $\mathrm{K}$ factor for the specific area.

\section{ACKNOWLEDGMENT}

Thanks to ICT-Robotics department in Science Technopark, Telecommunication Network Laboratory, and Multimedia Communication Laboratory for all the facilities and guidance that has been provided.

This research has been supported by the grant of Penelitian Dasar Unggulan Perguruan Tinggi 2021, the Ministry of Education, Culture, Research and Technology Republic of Indonesia.

\section{REFERENCES}

[1] K. Abboud, H. A. Omar and W. Zhuang, "Interworking of DSRC and cellular network technologies for V2X communications: a survey," IEEE Trans. Veh. Technol., vol. 65, no. 12, pp. 94579470, 2016.

[2] D. K. K. Vaishali and D. Khairnar, "Performance of vehicle-tovehicle communication using IEEE 802.11p in vehicular ad-hoc network environtment," International Journal of Network Security \& Its Application, vol. 5, no. 2, pp. 143-170, 2013.

[3] X. Shen, J. Li, J. Chen and S. He, "Heterogeneous LTE/DSRC approach to support real-time vehicular communications," in 10th International Conference on Advanced Infocomm Technology (ICAIT), Stockholm, 2018.

[4] P. Chyne, D. Kandar and B. S. Paul, "LTE-IEEE 802.11p HetNets interoperability for efficient vehicular communication," in 2017 IEEE International Conference on Power, Control, Signals and Instrumentation Engineering (ICPCSI), Chennai, 2017.

[5] L. Tytgat, O. Yaron, S. Pollin, I. Moerman and P. Demeester, "Avoiding collisions between IEEE 802.11 and IEEE 802.15.4 through coexistence aware clear channel assessment," EURASIP Journal on Wireless Communications and Networking, Vol. 1 22012-137, pp. 1-15, 2012.

[6] European Telecommunications Standards Institute, "Intelligent Transport Systems (ITS); Part 1: Road Hazard Signalling (RHS) application requirements specification," European Telecommunications Standards Institute, ETSI TS 101 539-1, 2013.

[7] V. Iordache, M. Minea and R. A. Gheorghiu, "Considerations for using ZigBee technology in vehicular non-critical applications," in 2017 Federated Conference on Computer Science and Information Systems (FedCSIS, Prague, 2017.

[8] V. Iordache, R. A. Gheorghiu, M. Minea and A. C. Cormos, "Field testing of Bluetooth and ZigBee technologies for vehicleto-infrastructure applications," in 2017 13th International Conference on Advanced Technologies, Systems and Services in Telecommunications (TELSIKS), Nis, 2017.

[9] European Telecommunications Standards Institute, "Intelligent Transport Systems (ITS); Vehicular Communications; Basic Set of Applications; Part 2: Specification of Cooperative Awareness Basic Service," European Telecommunications Standards Institute, ETSI TS 102 637-2 V1.2, 2011.

[10] V. Imandita and Muzayanah, "Analisis kebisingan dan volume kendaraan di jalan Ketintang kota Surabaya," Swara Bhumi, vol. 5, no. 61, pp. 193-199, 2018

[11] S. R. Saunders and A. Aragón-Zavala, Antennas and Propagation for Wireless Communication Systems, 2nd Ed., Chichester: John Wiley \& Sons, 2007.

[12] S. Medawar, P. Händel and P. Zetterberg, "Ricean K-factor estimation and investigation of urban wireless measurements," in 2012 IEEE International Conference on Wireless Information Technology and Systems (ICWITS), Maui, 2012.

[13] S. Zhu, et al., "Probability Distribution of Rician K-Factor in Urban, Suburban and Rural Areas Using Real-World Captured Data," IEEE Trans. Antennas and Propag., vol. 162, no. 7, pp. 3835-3839, 2014.

[14] P. Eamsomboon, P. Keeratiwintakorn and C. Mitrpant, "The performance of $\mathrm{Wi}-\mathrm{Fi}$ and Zigbee networks for inter-vehicle communication in Bangkok metropolitan area," in 2008 8th International Conference on ITS Telecommunications, Phuket, 2008 . 